\title{
Metrics with conic singularities and spherical polygons
}

\author{
Alexandre Eremenko, Andrei Gabrielov ${ }^{\dagger}$ and Vitaly Tarasov
}

September 25, 2018

\begin{abstract}
A spherical $n$-gon is a bordered surface homeomorphic to a closed disk, with $n$ distinguished boundary points called corners, equipped with a Riemannian metric of constant curvature 1, except at the corners, and such that the boundary arcs between the corners are geodesic. We discuss the problem of classification of these polygons and enumerate them in the case that two angles at the corners are not multiples of $\pi$. The problem is equivalent to classification of some second order linear differential equations with regular singularities, with real parameters and unitary monodromy.

MSC 2010: 30C20, 34M03.

Keywords: surfaces of positive curvature, conic singularities, Schwarz equation, accessory parameters, conformal mapping, circular polygons, Kostka numbers.
\end{abstract}

\section{Introduction}

Let $S$ be a compact Riemann surface, and $\left\{a_{0}, \ldots, a_{n-1}\right\}$ a finite set of points on $S$. Let us consider a conformal Riemannian metric on $S$ of constant curvature $K \in\{0,1,-1\}$ with conic singularities at the points $a_{j}$. This means that in a local conformal coordinate $z$ the length element of the metric

\footnotetext{
* Supported by NSF grant DMS-1351836.
}

†Supported by NSF grant DMS-1161629. 
is given by the formula $d s=\rho(z)|d z|$, where $\rho$ is a solution of the differential equation

$$
\Delta \log \rho+K \rho^{2}=0 \quad \text { in } \quad S \backslash\left\{a_{0}, \ldots, a_{n-1}\right\},
$$

and $\rho(z) \sim|z|^{\alpha_{j}-1}$, for the local coordinate $z$ which is equal to 0 at $a_{j}$. Here $\alpha_{j}>0$, and $2 \pi \alpha_{j}$ is the total angle around the singularity $a_{j}$.

Alternatively, for every point in $S$, there exists a local coordinate $z$ for which

$$
d s=\frac{2 \alpha|z|^{\alpha-1}|d z|}{1+K|z|^{2}}
$$

where $\alpha>0$. At the singular points $a_{j}$ we have $\alpha=\alpha_{j}$ while at all other points, $\alpha=1$.

In 1890, the Göttingen Mathematical Society proposed the study of equation (1.1) as a competition topic, probably by suggestion of H. A. Schwarz [30]. E. Picard wrote several papers on the subject, [24, 25, 26], see also [27. Chap. 4]. When $K<0$, the topic is closely related to the uniformization of orbifolds [30, 9, 28]. In the case of uniformization of orbifolds, one is interested in the angles $2 \pi \alpha_{j}=2 \pi / m_{j}$, where $m_{j}$ are positive integers. The case $K \leq 0$ is quite well understood, but very little is known on the case $K>0$.

McOwen [20] and Troyanov [34] studied the general question of existence and uniqueness of such metrics with prescribed $a_{j}, \alpha_{j}$ and $K$. Troyanov also considered the case of non-constant curvature $K$. One necessary condition that one has to impose on these data follows from the Gauss-Bonnet theorem: the quantity

$$
\chi(S)+\sum_{j=0}^{n-1}\left(\alpha_{j}-1\right) \quad \text { has the same sign as } K .
$$

Here $\chi$ is the Euler characteristic. Indeed, this quantity multiplied by $2 \pi$ is the integral curvature of the smooth part of the surface.

It follows from the results of Picard, McOwen and Troyanov, that for $K \leq 0$, condition (1.2) is also sufficient for the existence of the metric with conic singularities at arbitrary points $a_{j}$ and angles $2 \pi \alpha_{j}$. The metric with given $a_{j}$ and $\alpha_{j}$ is unique when $K<0$, and unique up to a constant multiple when $K=0$.

In the case of positive curvature, the results are much less complete. The result of Troyanov that applies to $K=1$ is the following: 
Let $S$ be a compact Riemann surface, $a_{0}, \ldots, a_{n-1}$ points on $S$, and $\alpha_{0}, \ldots, \alpha_{n-1}$ positive numbers satisfying

$$
0<\chi(S)+\sum_{j=0}^{n-1}\left(\alpha_{j}-1\right)<2 \min \left\{1, \alpha_{0}, \ldots, \alpha_{n-1}\right\} .
$$

Then there exists a conformal metric of positive curvature 1 on $S$ with conic singularities at $a_{j}$ and angles $2 \pi \alpha_{j}$.

F. Luo and G. Tian [19] proved that if the condition $0<\alpha_{j}<1$ is satisfied for $0 \leq j \leq n-1$, then (1.3) is necessary and sufficient, and the metric with given $a_{j}$ and $\alpha_{j}$ is unique.

In general, the second inequality in (1.3) is not necessary. In this paper, we only consider the simplest case when $S$ is the sphere, so $\chi(S)=2$. The problem of description and classification of conformal metrics of curvature 1 with conic singularities on the sphere has applications to the study of certain surfaces of constant mean curvature [10, 2, 3], and to several other questions of geometry and physics [16, 34].

The so-called "real case" is interesting and important. Suppose that all singularities belong to a circle on the sphere $S$, and we only consider the metrics which are symmetric with respect to this circle. Then the circle splits $S$ into two symmetric halves, and each of them is a spherical polygon, for which we state a formal definition:

Definition 1.1 A spherical $n$-gon is a closed disk with $n$ distinguished boundary points $a_{j}$ called the corners, equipped with a conformal Riemannian metric of constant curvature 1 everywhere except the corners, and such that the sides (boundary arcs between the corners) are geodesic. The metric has conic singularities at the corners.

Example 1.2 Let us consider flat $n$-gons, which are defined similarly, with $K=0$. The necessary and sufficient condition for the existence of a flat $n$-gon with angles $\pi \alpha_{j}$ is given by the Gauss-Bonnet theorem which in this case says that

$$
\sum \alpha_{j}=n-2
$$

and the polygon with given angles and prescribed corner 1 is unique up to a scaling factor. The simplest proof of these facts is the Schwarz-Christoffel

\footnotetext{
${ }^{1}$ By this we mean that the images of the corners under a conformal map onto a disk are prescribed.
} 
formula. Thus our subject can be considered as a generalization of the SchwarzChristoffel formula to the case of positive curvature.

In [36, 4], all possibilities for spherical triangles are completely described, see also [10] where a minor error in [4, Theorem 2] is corrected. In the case of triangles, the metric is uniquely determined by the angles in the case that none of the $\alpha_{j}$ is an integer.

The case when all $\alpha_{j}$ are integers, and $n$ is arbitrary, is also well understood. In this case, the line element of the metric has the global representation

$$
d s=\frac{2\left|f^{\prime}\right||d z|}{1+|f|^{2}},
$$

where $f$ is a rational function. The singular points $a_{j}$ are the critical points of $f, \alpha_{j}-1$ is the multiplicity of the critical point at $a_{j}$, and $\alpha_{j}$ is the local degree of $f$ at $a_{j}$.

Thus the problem with all integer $\alpha_{j}$ is equivalent to describing rational functions with prescribed critical points $[12,31,5,6,6,8,8$. Also, this problem is equivalent to diagonalization of commuting Hamiltonians for the quantum Gaudin model by the Bethe ansatz method [11, 21, 23].

Almost nothing is known in the case that some of the $\alpha_{j}$ are not integers, the number of singularities is greater than 3 , and the second inequality in (1.3) is violated.

In this paper we investigate the case when two of the $\alpha_{j}$ are non-integer while the rest are integers, with the emphasis on the real case. See also [1] where some examples of such metrics are given.

Our method can be considered a generalization of the method in [5, 6, 8] and Klein [17], who classified circular triangles with arbitrary angles, not necessarily geodesic. A modern paper which uses Klein's approach to triangles is [37.

The contents of the paper is the following. In section 2 we recall the connection of the problem with linear differential equations. In section 3 we describe what is known about the case when all $\alpha_{j}$ are integers, with a special emphasis on the "real case" when all $a_{j}$ belong to the real line.

In sections 446 we study the case when two of the $\alpha_{j}$ are not integers and the others are integers, and give a complete classification of spherical polygons in this case. 


\section{Connection with linear differential equa- tions}

Let $(S, d s)$ be the Riemann sphere equipped with a metric with conic singularities. Every smooth point of $S$ has a neighborhood which is isometric to a region on the standard unit sphere $\mathbf{S}$; let $f$ be such an isometry. Then $f$ has an analytic continuation along every path in $S \backslash\left\{a_{0}, \ldots, a_{n-1}\right\}$, and we obtain a multi-valued function which is called the developing map. The monodromy of $f$ consists of orientation-preserving isometries (rotations) of S, so the Schwarzian derivative

$$
F(z):=\frac{f^{\prime \prime \prime}}{f^{\prime}}-\frac{3}{2}\left(\frac{f^{\prime \prime}}{f^{\prime}}\right)^{2}
$$

is a single valued function.

Developing map is completely characterized by the properties that it has an analytic continuation along any curve in $S \backslash\left\{a_{0}, \ldots, a_{n-1}\right\}$, has asymptotics $\sim c\left(z-a_{j}\right)^{\alpha_{j}}$ as $z \rightarrow a_{j}$, where $c \neq 0$, and has $\operatorname{PSU}(2)=S O(3)$ monodromy. It is possible that two such maps with the same $a_{j}$ and $\alpha_{j}$ are related by post-composition with a fractional-linear transformation. The metrics arising from such maps will be called equivalent. Following [10, we say that the metric is reducible if its monodromy group is commutative (which is equivalent to all monodromy transformations having a common fixed point). In the case of irreducible metrics, each equivalence class contains only one metric. For reducible metrics, the equivalence class is a 1parametric family when the monodromy is non-trivial and a 2-parametric family when monodromy is trivial.

The asymptotic behavior of $f$ at the singular points $a_{j}$ implies that the only singularities of $F$ on the sphere are double poles, so $F$ is a rational function, and we obtain the Schwarz differential equation (2.1) for $f$.

It is well-known that the general solution of the Schwarz differential equation is a ratio of two linearly independent solutions of the linear differential equation

$$
y^{\prime \prime}+P y^{\prime}+Q y=0, \quad f=y_{1} / y_{0},
$$

where

$$
F=-P^{\prime}-P^{2} / 2+2 Q
$$

For example one can take $P=0$, then $Q=F / 2$. Another convenient choice is to make all poles but one of $P$ and $Q$ simple. When $n=3$, equation (2.2) 
is equivalent to the hypergeometric equation, and when $n=4$ to the Heun equation [29].

The singular points $a_{j}$ of the metric are the singular points of the equation (2.2). These singular points are regular, and to each point correspond two exponents $\alpha_{j}^{\prime}>\alpha_{j}^{\prime \prime}$, so that $\alpha_{j}=\alpha_{j}^{\prime}-\alpha_{j}^{\prime \prime}$. If $\alpha_{j}$ is an integer for some $j$, we have an additional condition of the absence of logarithms in the formal solution of (2.2) near $a_{j}$.

It is easy to write down the general form of a Fuchsian equation with prescribed singularities and prescribed exponents at the singularities. By a fractional-linear change of the independent variable one can achieve that one singular point, say $a_{n-1}$ is $\infty$. Then by changes of the variable $y(z) \mapsto$ $y(z)\left(z-a_{j}\right)^{\beta_{j}}$, one can achieve that the smaller exponent at each finite singular point is 0 , see [29]. After a normalization, $n-3$ parameters remain, the so-called accessory parameters $[13$, Ch. IV, 7]

$$
w^{\prime \prime}+\sum_{j=0}^{n-2} \frac{1-\alpha_{j}}{z-a_{j}} w^{\prime}+\frac{\alpha^{\prime} \alpha^{\prime \prime} z^{n-3}+\lambda_{n-4} z^{n-4}+\ldots+\lambda_{0}}{\prod_{j=0}^{n-2}\left(z-a_{j}\right)} w=0,
$$

where

$$
\alpha_{n-1}=\alpha^{\prime}-\alpha^{\prime \prime}, \quad \sum_{j=0}^{n-2} \alpha_{j}+\alpha^{\prime}+\alpha^{\prime \prime}=n-2 .
$$

Here the exponents at the singular points are described by the Riemann symbol

$$
P\left\{\begin{array}{cccccc}
a_{0} & a_{1} & a_{2} & \ldots & \infty & \\
0 & 0 & 0 & \ldots & \alpha^{\prime \prime} & ; z \\
\alpha_{0} & \alpha_{1} & \alpha_{2} & \ldots & \alpha^{\prime}
\end{array}\right\}
$$

The first line lists the singularities, the second the smaller exponents and the third the larger exponents. So the angle at infinity is $\alpha_{n-1}=\alpha^{\prime}-\alpha^{\prime \prime}$. The accessory parameters are $\lambda_{0}, \ldots, \lambda_{n-4}$.

To obtain a conformal metric of curvature 1, one has to choose these accessory parameters in such a way that the monodromy group of the equation is conjugate to a subgroup of $P S U(2)$.

Solving (2.4), we obtain

$$
\alpha^{\prime}=\frac{1}{2}\left(n-2+\alpha_{n-1}-\sum_{j=0}^{n-2} \alpha_{j}\right)
$$


and

$$
\alpha^{\prime \prime}=\frac{1}{2}\left(n-2-\alpha_{n-1}-\sum_{j=0}^{n-2} \alpha_{j}\right) .
$$

The question of existence of a spherical metric with given singularities $a_{0}, a_{1}, \ldots, a_{n-2}, \infty$ and given angles $2 \pi \alpha_{j}, 0 \leq j \leq n-1$ is equivalent to the following: When one can choose the accessory parameters $\lambda_{j}$ so that the projective monodromy group of the equation (2.3) is conjugate to a subgroup of $P S U(2)$ ?

The necessary condition (1.2) can be restated for the equation (2.3) as

$$
\alpha^{\prime \prime}<0
$$

For the case of four singularities, questions similar to our problem were investigated in [18, 14, 32, 33]: when can one choose the accessory parameter so that the monodromy group of the Heun equation preserves a circle? All these authors consider the problem under the assumption

$$
0 \leq \alpha_{j}<1, \quad \text { for } \quad 0 \leq j \leq n-1
$$

In the paper [3] the problem of choosing the accessory parameter so that the monodromy group is conjugate to a subgroup in $P S U(2)$ is discussed. However all results of this paper are also proved only under the assumption (2.7). Assumption (2.7) seems to be essential for the methods of Klein [18, Hilb [14], Smirnov [32, 33], Dorfmeister and Schuster [3, 2].

\section{Case when all $\alpha_{j}$ are integers}

If all $\alpha_{j}$ are integers, the developing map $f$ is a rational function, and the metric of curvature 1 with conic singularities can be globally described as the pull-back of the spherical metric via $f$, that is

$$
d s=\frac{2\left|f^{\prime}\right||d z|}{1+|f|^{2}} .
$$

The singular points of the metric are critical points of $f$, and $\alpha_{j}-1$ are multiplicities of these critical points.

The following results are known for this case. 
First of all, the sum of $\alpha_{j}-1$ must be even: if $d$ is the degree of $f$, then

$$
2+\sum_{j=1}^{n}\left(\alpha_{j}-1\right)=2 d .
$$

This is stronger than the general necessary condition (1.2).

Second,

$$
\alpha_{j} \leq d \text { for all } j,
$$

because a rational function of degree $d$ cannot have a point where the local degree is greater than $d$.

Subject to these two restrictions (3.1) and (3.2), a rational function with prescribed critical points at $a_{j}$ of multiplicities $\alpha_{j}-1$ always exists [12, 31]. Thus for every $a_{j}$ and every $\alpha_{j}$ satisfying (3.1) and (3.2) there exist metrics of curvature 1 on $S$ with angles $2 \pi \alpha_{j}$ at $a_{j}$.

Two rational functions $f_{1}$ and $f_{2}$ are called equivalent if $f_{1}=\phi \circ f_{2}$, where $\phi$ is a fractional-linear transformation. Equivalent functions have the same critical points with the same multiplicities. Equivalent functions generate equivalent metrics.

The number of equivalence classes of rational functions with prescribed critical points and multiplicities is at most $\mathbf{K}\left(\alpha_{0}-1, \ldots, \alpha_{n-1}-1\right)$, where $\mathbf{K}$ is the Kostka number which can be described as follows. Consider Young diagrams of shape $2 \times(d-1)$. They consist of two rows of length $d-1$ one above another. A semi-standard Young tableau (SSYT) is a filling of such a diagram with positive integers such that an integer $k$ appears $m_{k-1}$ times, the entries are strictly increasing in the columns and non-decreasing in the rows. The number of such SSYT's is the Kostka number $\mathbf{K}\left(m_{0}, \ldots, m_{n-1}\right)$.

For a generic choice of the critical points $a_{j}$, the number of classes of rational functions is equal to the Kostka number, see [31, 8].

Suppose now that the points $a_{j}$ and the corresponding multiplicities $\alpha_{j}$ are symmetric with respect to some circle. We may assume without loss of generality that this circle is the real line $\mathbf{R} \cup\{\infty\}$. It may happen that among the rational functions $f$ with these given critical points and multiplicities none are symmetric. So the resulting metrics are all asymmetric as well [6, 7].

However, there is a surprising result [5, 6, 8, 21] which was conjectured by B. and M. Shapiro:

If all critical points of a rational function lie on a circle, then the function is equivalent to a function symmetric with respect to this circle. Moreover, 
in this case there are exactly $\mathbf{K}$ equivalence classes of rational functions with prescribed critical points.

It is interesting to find out which of these results can be extended to the general case of arbitrary positive $\alpha_{j}$.

\section{The case of two non-integer $\alpha_{j}$. Condition on the angles.}

In the rest of the paper we study the case with two non-integer $\alpha_{j}$. Some examples of polygons with this property can be found in [1]. We answer the following questions:

a) In the equation (2.3), for which $\alpha_{j}$ one can choose the accessory parameters so that the monodromy group is conjugate to a subgroup of $P S U(2)$ ?

b) If $\alpha_{j}$ satisfy a), how many choices of accessory parameters satisfying a) are possible?

c) If in addition all $a_{j}$ are real, how many choices of real accessory parameters satisfying a) are possible?

One cannot have exactly one non-integer $\alpha_{j}$. Indeed, in this case the developing map $f$ would have just one branching point on the sphere, which is impossible by the Monodromy theorem.

Let us consider the case of two non-integer angles. In this section we obtain a necessary and sufficient condition on the angles for this case, that is solve problem a).

We place the two singularities corresponding to non-integer $\alpha$ at $a_{0}=0$ and $a_{n-1}=\infty$, and let the total angles at these points be $2 \pi \alpha_{0}$ and $2 \pi \alpha_{n-1}$, where $\alpha_{0}$ and $\alpha_{n-1}$ are not integers. Then the developing map has an analytic continuation in $\mathbf{C}^{*}$ from which we conclude that the monodromy group must be a cyclic group generated by a rotation $z \mapsto z e^{2 \pi i \alpha}$, with some $\alpha \in(0,1)$. This means that $f(z)$ is multiplied by $e^{2 \pi i \alpha}$ when $z$ traverses a simple loop around the origin. Thus $g(z)=z^{-\alpha} f(z)$ is a single valued function with at most power growth at 0 and $\infty$. Then we have a representation $f(z)=$ $z^{\alpha} g(z)$, where $g$ is a rational function. Then $\alpha_{0}=|k+\alpha|, \alpha_{n-1}=|j+\alpha|$, where $k$ and $j$ are integers, so either $\alpha_{0}-\alpha_{n-1}$ or $\alpha_{0}+\alpha_{n-1}$ is an integer. The angles at the other singular points of the metric are integer multiples of $2 \pi$, and they are the critical points of $f$ other than 0 and $\infty$.

Let $g=P / Q$ where $P$ and $Q$ are polynomials without common zeros of 
degrees $p, q$ respectively. Let $p_{0}, q_{0}$ be the multiplicities of zeros of $P$ and $Q$ at 0 . Then $\min \left\{p_{0}, q_{0}\right\}=0$, because we may assume that the fraction $P / Q$ is irreducible.

The equation for the critical points of $f$ is the following:

$$
z\left(P^{\prime}(z) Q(z)-P(z) Q^{\prime}(z)\right)+\alpha P(z) Q(z)=0 .
$$

Let $2 \pi \alpha_{0}, 2 \pi \alpha_{1} \ldots 2 \pi \alpha_{n-1}$ be the angles at the conical singularities at $0, a_{1}, \ldots, a_{n-2}, \infty$, so that $\alpha_{j} \geq 2$ are integers for $1 \leq j \leq n-2$.

Denote

$$
\sigma:=\alpha_{1}+\ldots+\alpha_{n-2}-n+2 \geq 0 .
$$

Then we have the following system of equations

$$
\begin{aligned}
\alpha_{0} & =\left|p_{0}-q_{0}+\alpha\right| \\
\sigma & =p+q-\max \left\{p_{0}, q_{0}\right\} \\
\alpha_{\infty} & =|p-q+\alpha| .
\end{aligned}
$$

The first and the last equations follow immediately from the representation $f(z)=z^{\alpha} P(z) / Q(z)$ of the developing map. The second equation holds because the left hand side of (4.1) is a polynomial of degree exactly $m+n$, therefore the sum of multiplicities of its zeros at $a_{j}$ for $1 \leq j \leq n-2$ must be $p+q-\max \left\{p_{0}, q_{0}\right\}$

Solving this system of equations (4.2) in non-negative integers satisfying $\min \left\{p_{0}, q_{0}\right\}=0, p_{0} \leq p, q_{0} \leq q$, we obtain necessary and sufficient conditions the angles should satisfy, which we state as

Theorem 4.1 Suppose that $n$ points $a_{0}, \ldots, a_{n-1}$ on the Riemann sphere and numbers $\alpha_{j}>0,0 \leq j \leq n-1$, are such that $\alpha_{j} \geq 2$ are integers for $1 \leq j \leq n-2$.

The necessary and sufficient conditions for the existence of a metric of curvature 1 on the sphere, with conic singularities at $a_{j}$ and angles $2 \pi \alpha_{j}$ are the following:

a) If $\sigma+\left[\alpha_{0}\right]+\left[\alpha_{\infty}\right]$ is even, then $\alpha_{0}-\alpha_{\infty}$ is an integer, and

$$
\left|\left[\alpha_{0}\right]-\left[\alpha_{\infty}\right]\right| \leq \sigma .
$$

b) If $\sigma+\left[\alpha_{0}\right]+\left[\alpha_{\infty}\right]$ is odd, then $\alpha_{0}+\alpha_{\infty}$ is an integer, and

$$
\left[\alpha_{0}\right]+\left[\alpha_{\infty}\right]+1 \leq \sigma
$$


Proof. Let us first verify that conditions a)-b) are necessary and sufficient for the existence of a unique solution $p, q, p_{0}, q_{0}, \alpha$ of the system (4.2) satisfying $\min \left\{p_{0}, q_{0}\right\}=0, \quad p_{0} \leq p, \quad q_{0} \leq q, \quad \alpha \in(0,1)$.

We consider four cases.

Case 1. $p \geq q, m_{0} \geq q_{0}=0$. Then

$$
\begin{aligned}
p_{0} & =\left[\alpha_{0}\right], \\
p+q & =\sigma+\left[\alpha_{0}\right], \\
p-q & =\left[\alpha_{\infty}\right] .
\end{aligned}
$$

So $\alpha_{0}-\alpha_{\infty}$ is an integer, and $2 p=\sigma+\left[\alpha_{0}\right]+\left[\alpha_{\infty}\right]$ is even. Now

$$
\begin{gathered}
0 \leq 2\left(p-p_{0}\right)=\sigma-\left[\alpha_{0}\right]+\left[\alpha_{\infty}\right], \\
0 \leq 2 q=\left(\sigma+\left[\alpha_{0}\right]-\left[\alpha_{\infty}\right]\right),
\end{gathered}
$$

so we obtain (4.3).

Case 2. $p \geq q, q_{0}>p_{0}=0$. Then

$$
\begin{aligned}
q_{0} & =\left[\alpha_{0}\right]+1, \\
p+q & =\sigma+\left[\alpha_{0}\right]+1, \\
p-q & =\left[\alpha_{\infty}\right] .
\end{aligned}
$$

So $\alpha_{0}+\alpha_{\infty}$ is an integer, and $2 p=\sigma+\left[\alpha_{0}\right]+\left[\alpha_{\infty}\right]+1$ is even. We have $2 q=\left(\sigma+\left[\alpha_{0}\right]-\left[\alpha_{\infty}\right]+1\right.$, so

$$
0 \leq 2\left(q-q_{0}\right)=\sigma-\left[\alpha_{0}\right]-\left[\alpha_{\infty}\right]-1 .
$$

which gives (4.4).

Case 3. $p<q, p_{0} \geq q_{0}=0$. Then

$$
\begin{aligned}
p_{0} & =\left[\alpha_{0}\right], \\
p+q & =\sigma+\left[\alpha_{0}\right], \\
q-p & =\left[\alpha_{\infty}\right]+1 .
\end{aligned}
$$

So $\alpha_{0}+\alpha_{\infty}$ is an integer, and $2 q=\sigma+1+\left[\alpha_{0}\right]+\left[\alpha_{\infty}\right]$ is even. We have $2 p=\sigma-1+\left[\alpha_{0}\right]-\left[\alpha_{\infty}\right]$, so

$$
0 \leq 2\left(p-p_{0}\right)=\sigma-1-\left[\alpha_{0}\right]-\left[\alpha_{\infty}\right],
$$


which is (4.4).

Case 4. $p<1, q_{0}>p_{0}=0$. Then

$$
\begin{aligned}
q_{0} & =\left[\alpha_{0}\right]+1, \\
p+q & =\sigma+1+\left[\alpha_{0}\right], \\
q-p & =\left[\alpha_{\infty}\right]+1 .
\end{aligned}
$$

So $\alpha_{0}-\alpha_{\infty}$ is an integer, and $2 q=\sigma+\left[\alpha_{0}\right]+\left[\alpha_{\infty}\right]+2$ is even. We have $0 \leq 2 p=\sigma+\left[\alpha_{0}\right]-\left[\alpha_{\infty}\right]$, and

$$
0 \leq 2\left(q-q_{0}\right)=\sigma-\left[\alpha_{0}\right]+\left[\alpha_{\infty}\right]
$$

which implies (4.3).

Thus the conditions a) and b) are necessary.

Now we set

$$
P(z)=z^{\max \left\{p_{0}, q_{0}\right\}} \prod_{j=1}^{n-2}\left(z-a_{j}\right)^{\alpha_{j}-1} .
$$

Second equation in (4.2) gives $\operatorname{deg} P=p+q$. Now we consider the equation

$$
z\left(P^{\prime} Q-P Q^{\prime}\right)+\alpha P Q=R .
$$

This equation must be solved in polynomials $P, Q$ of degrees $p, q$ having roots of multiplicities $p_{0}, q_{0}$ at 0 . Non-zero polynomials of degree at most $m$ modulo proportionality can be identified with points of the complex projective space $\mathbf{P}^{m}$. The map

$$
W_{\alpha}: \mathbf{P}^{p} \times \mathbf{P}^{q} \rightarrow \mathbf{P}^{p+q}, \quad(P, Q) \mapsto z\left(P^{\prime} Q-P Q^{\prime}\right)+\alpha P Q
$$

is well defined. It is a finite map between compact algebraic varieties, and it can be represented as a linear projection of the Veronese variety. Its degree is known: it is equal to

$$
\left(\begin{array}{c}
p+q \\
p
\end{array}\right)
$$

Thus the equation (4.5) always has a complex solution $(P, Q)$. The function $f=z^{\alpha}(P / Q)$ is then a developing map with the required properties. So conditions a)-b) are sufficient. This completes the proof of the first statement.

We will later prove that for generic polynomial $R$ there are exactly (4.7) preimages under the map $W_{\alpha}$. 


\section{Case $\alpha=1 / 2$}

Let us consider a spherical polygon, parametrized by the upper half-plane $H$, with corners

$$
0=a_{0}<a_{1}<\ldots<a_{n-2}, \quad a_{n-1}=\infty
$$

and suppose that the angles $\pi \alpha_{j}$ at $a_{j}$ are integer multiples of $\pi$ for $1 \leq j \leq$ $n-2$ while $2 \alpha_{0}$ and $2 \alpha_{n-1}$ are odd integers. This means that $\alpha=1 / 2$ in (4.6), and the roots of the polynomial (4.1) are non-negative. In intrinsic terms, condition (5.1) means that the corners with non-integer angles of our spherical polygon are adjacent.

Let $Q$ be our spherical polygon, and $Q^{\prime}$ its mirror image. We paste $Q$ and $Q^{\prime}$ together identifying the sides between the non-integer corners isometrically. The result is a polygon $Q^{*}$ with $2 n-2$ corners and all integer angles. With our half-plane model this procedure can be performed in the following way. Parametrize our original polygon $Q$ by the first quadrant, with corners as in (5.1), and $Q^{\prime}$ by the second quadrant with corners at

$$
0=a_{0}>-a_{1}>-a_{2}>\ldots>-a_{n-2}>-a_{n-1}=\infty
$$

Then the upper half-plane will parametrize $Q^{*}$. Thus the developing map $f^{*}: H \rightarrow \mathbf{S}$ of the polygon $Q^{*}$ is a real rational function with all critical points real. It satisfies

$$
f^{*}(-\bar{z})=-\overline{f^{*}(z)}
$$

so $f^{*}$ is odd. In the opposite direction, if $f^{*}$ is an odd real rational function with with all critical points real, then the restriction of

$$
f(z)=f^{*}(\sqrt{z})
$$

to $H$ parametrizes a spherical polygon of the considered type.

Thus we obtain

Proposition 5.1 Equivalence classes of spherical polygons with all angles but two integer, and adjacent non-integer angles such that $2 \alpha_{0}$ and $2 \alpha_{n}$ are odd integers, are in one-to-one correspondence with classes of odd real rational functions with real critical points. The correspondence is explicitly given by (5.3). 
We recall that equivalence classes of real rational functions with real critical points are counted by chord diagrams [5, 6, 8]. Let $g$ be such a function. Then $g^{-1}(\mathbf{R} \cup \infty)$ restricted to $H$ is a chord diagram: it consists of smooth arcs in $H$ with ends on $\mathbf{R} \cup\{\infty\}$, and these arcs are disjoint except at the ends. These ends are the critical points. At a critical point of multiplicity $m, m$ arcs meet, so in our case we have

$$
m_{0}=2 \alpha_{0}-1, \quad m_{n-1}=2 \alpha_{n-1}-1, \quad m_{j}=\alpha_{j}-1, \quad 1 \leq j \leq n-2
$$

for the critical points on the ray $0 \leq x \leq \infty$. A rational function is odd iff the diagram is invariant with respect to $z \mapsto-\bar{z}$, and even number of arcs meet at 0 and at $\infty$. We call such chord diagrams odd.

Without these conditions, the number $\mathbf{K}\left(n_{1}, \ldots, n_{k}\right)$ of all chord diagrams with vertices of orders $n_{1}, \ldots, n_{k}$ is the Kostka number which was defined in section 3. A necessary condition for existence of such a diagram is that $n_{1}+\ldots+n_{k}$ is an even number $2 d-2$. We set $\mathbf{K}\left(n_{1}, \ldots, n_{k}\right)=0$ if the SSYT with parameters $n_{j}$ in the definition of Kostka number does not exist. We recall that Kostka number does not change after a permutation of $n_{j}$.

Now we return to odd chord diagrams. Let $m_{0}, m_{1}, \ldots, m_{n-1}$ be the multiplicities of critical points on $0 \leq x \leq \infty$, and

$E\left(m_{0}, m_{1}, \ldots, m_{n-2}, m_{n-1}\right)$ the number of chord diagrams corresponding to odd rational functions with critical points of multiplicities $m_{0}, \ldots, m_{n-1}$ on $0 \leq x \leq \infty$. We express this quantity in terms of Kostka numbers.

\section{Theorem 5.2}

$$
E\left(m_{0}, m_{1}, \ldots, m_{n-2}, m_{n-1}\right)=\mathbf{K}\left(r, m_{1}, \ldots, m_{n-2}, s\right),
$$

where positive integers $r$ and s satisfy

$$
r+s>m_{1}+\ldots+m_{n-2},
$$

and are defined as follows:

If $\mu:=\left(m_{0}+m_{n-1}\right) / 2+m_{1}+\ldots+m_{n-2}$ is even, then $r=m_{0} / 2+k$ and $s=m_{n-1} / 2+k$, where $k$ is large enough to satisfy (5.5).

If $\mu$ is odd, then $r=\left|m_{0}-m_{n-1}\right| / 2+k+1$ and $s=k$, where $k$ is large enough to satisfy (5.5).

Proof. Let us say that an edge of a chord diagram is crossing if its one endpoint is positive and another negative. The number $\nu$ of crossing edges satisfies $\nu \equiv \mu \bmod 2$. 
If $\nu$ is even, we have

$$
E\left(m_{0}, m_{1}, \ldots, m_{n-2}, m_{n-1}\right)=E\left(m_{0}+2, m_{1}, \ldots, m_{n-2}, m_{n-1}+2\right) .
$$

Indeed we can establish a bijection between the nets counted by both sides of (5.6) as follows. If $\nu=0$ we can add two edges connecting $a_{0}=0$ and $a_{n-1}=\infty$. If $\nu \neq 0$, we can replace two extreme crossing edges (closest to $a_{0}$ and $\left.a_{n-1}\right)$ by the edges going to $a_{0}$ and $a_{n-1}$, without changing the other endpoints of these edges. Notice that these operations do not change the parity of $\nu$.

If $\nu$ is odd, and $m_{n-1}>0$, we have

$$
E\left(m_{0}, m_{1}, \ldots, m_{n-2}, m_{n-1}\right)=E\left(m_{0}+2, m_{1}, \ldots, m_{n-2}, m_{n-1}-2\right) .
$$

Indeed, in this case we can shift one crossing edge so that it becomes an edge ending at $a_{0}$, the other endpoint unchanged, and at $a_{n-1}$ to perform the opposite operation: replace one edge ending at $a_{n-1}$ by a crossing edge.

Similarly if $\nu$ is odd and $m_{0}>0$, we have

$$
E\left(m_{0}, m_{1}, \ldots, m_{n-2}, m_{n-1}\right)=E\left(m_{0}-2, m_{1}, \ldots, m_{n-2}, m_{n-1}+2\right) .
$$

These operations do not change the number $\nu$. Finally, if $\nu$ is odd and $m_{0}=0$, we have

$$
E\left(0, m_{1}, \ldots, m_{n-2}, m_{n-1}\right)=E\left(0, m_{1}, \ldots, m_{n-2}, m_{n-1}+2\right),
$$

and similarly, if $\nu$ is odd and $m_{n-1}=0$ then

$$
E\left(m_{0}, m_{1}, \ldots, 0\right)=E\left(m_{0}+2, m_{1}, \ldots, m_{n-2}, 0\right) .
$$

and this operation replaces $\nu$ by $\nu-1$, so it switches the parity of $\nu$.

Finally we notice that if $m_{0}>2\left(m_{1}+\ldots+m_{n-2}\right)$ then there can be no crossing edges, thus our chord diagram is simply the union of two symmetrical chord diagrams, and thus

$$
E\left(m_{0}, \ldots, m_{n-1}\right)=\mathbf{K}\left(m_{0} / 2, m_{1}, \ldots, m_{n-2}, m_{n-1} / 2\right) .
$$

The result follows from (5.6), (5.7), (5.8) and (5.9).

There is no explicit formula for Kostka numbers. But we need a simple formula in the generic case. We recall that the number $\mathbf{K}(1,1, \ldots, 1)$ of chord diagrams with $2 d$ vertices and one chord ending at each vertex is

$$
C_{d}=\frac{1}{d+1}\left(\begin{array}{c}
2 d \\
d
\end{array}\right)
$$

the Catalan number. 
Proposition 5.3 For $m$ vertices on the positive ray, one edge ending at each vertex, we have

$$
E_{m}:=E(0,1, \ldots, 1,0)=\left(\begin{array}{c}
m \\
{[m / 2]}
\end{array}\right)
$$

Proof. In view of Theorem 5.2 it is sufficient co count $\mathbf{K}\left(a, m_{0}, \ldots, m_{n-2}, b\right)$ with

$$
a+b>m_{1}+\ldots+m_{n-2} .
$$

Let us define the positive integer $d$ by the formula

$$
2(d-1)=a+b+m_{1}+\ldots+m_{n-2} .
$$

Then we are counting Young tableaux of shape $2 \times(d-1)$. In such a tableau, 1 's must stand in the first row on the left and $n$ 's in the second row on the right. In view of the inequality (5.10) the part of the tableau with entries $2, \ldots, n-1$ consists of two rows that have no common columns. the number of ways to fill these rows is the binomial coefficient.

Now we return to solution of the equation (4.5) with generic real $R$, whose roots are simple and positive, and $\operatorname{deg} R=p+q$. We conclude that $p=q$ or $q=p+1$, and obtain that the degree of the map $W_{\alpha}$ is

$$
\left(\begin{array}{c}
p+q \\
p
\end{array}\right)
$$

according to (4.7). So all preimages $W_{1 / 2}^{-1}(R)$ are real. Taking limits of such polynomials we conclude that

Proposition 5.4 For a real polynomial $R$ with non-negative roots, all solutions of the equation $W_{1 / 2}(P, Q)=R$ are real.

This proposition can be also derived from [22, Theorem 4.2 (ii)].

\section{Deformation argument}

Now we extend the results of the previous paragraph to the case of arbitrary $\alpha$ and prove the following 
Theorem 6.1 Let $\alpha_{0}, \alpha_{1}, \ldots, \alpha_{n-1}, n \geq 4$, be given positive numbers of which $\alpha_{0}$ and $\alpha_{n-1}$ are not integers and the rest are integers, and suppose that conditions of Theorem 4.1 are satisfied.

Then for given $a_{0}, \ldots, a_{n-1}$ there are at most

$$
E\left(2\left[\alpha_{0}\right]-1, \alpha_{1}-1, \ldots, \alpha_{n-2}-1,2\left[\alpha_{n-1}\right]-1\right)
$$

equivalence classes of metrics with conic singularities $a_{0}, \ldots, a_{n}$, and angles $\pi \alpha_{0}, \ldots, \pi \alpha_{n-1}$. For generic $a_{j}$ we have equality.

Moreover, we always have equality, when the $a_{j}$ lie on a circle in the cyclic order $a_{0}, \ldots, a_{n-1}$.

Let us fix some $\alpha \in(0,1)$. Let $Q$ be a spherical $n$-gon with all angles but two integer multiples of $\pi$, the two corners with non-integer angles adjacent, and the non-integer angles satisfy that $2 \alpha_{0}$ and $2 \alpha_{n-1}$ are odd integers.

We parametrize $Q$ by the upper half-plane $H$ as before, with corners as in (5.1). Let $f: Q \rightarrow \mathbf{S}$ be the developing map. Then all sides of $Q$ are mapped into two circles, $f\left(\mathbf{R}_{+}\right) \subset C_{0}$ and $f\left(\mathbf{R}_{-}\right) \subset C_{1 / 2}$. We may assume that $C_{0}$ is the real line and $C_{1 / 2}$ is the imaginary line.

Let $\psi: \mathbf{S} \rightarrow \mathbf{S}$ be a quasiconformal homeomorphism that leaves the real line pointwise fixed and maps the imaginary line onto $C_{\alpha}=\left\{r e^{i \alpha}: r \in \mathbf{R}\right\}$. Then

$$
g=\psi \circ f
$$

is a topologically holomorphic map $H \rightarrow \mathbf{S}$ which is a local homeomorphism in the interior of $H$ and maps the real line into $\mathbf{R} \cup C_{\alpha} \cup \infty$. By solving a Beltrami equation, we find a quasiconformal homeomorphism $\psi: H \rightarrow H$ such that

$$
g=\psi \circ f \circ \phi
$$

is holomorphic. Then $f: H \rightarrow \mathbf{S}$ is a developing map of a polygon $Q_{\alpha}$.

This correspondence can be inverted: for every spherical polygon $Q_{\alpha}$ with all angles but two integers, and adjacent corners with non-integer angles, and developing map $g$ we can find a polygon $Q$ whose non-integer angle at one vertex has fractional part $1 / 2$, such that the developing maps of $Q$ and $Q_{\alpha}$ are related by formula (6.3).

Let

$$
0<a_{1}<\ldots<a_{n-2}<\infty
$$

be the corners of $Q$, and $0<b_{1}<\ldots<b_{n-2}<\infty$ be the corners of $Q_{\alpha}$. We evidently have $a_{j}=\phi\left(b_{j}\right)$. It is convenient no normalize $a_{1}=b_{1}=1$, then 
the map $\psi$ which will uniquely define $\phi$. With this normalization, the set of possible configurations $a_{j}$ forms the interior of a simplex $\Delta_{a}$ of dimension $n-3$.

Let $N$ be the chord diagram corresponding to $f$ as in the previous section. It is known [8] that for a fixed chord diagram, and for each point $x \in \Delta_{a}$, there exists a unique equivalence class of rational functions and thus a map $f$. To this $f$ we put into correspondence the map $g$ by formula (6.3), and obtain a point $y=\Phi(x) \in \Delta_{b}$.

We claim that the map $\Phi$ is a diffeomorphism of open simplexes $\Delta_{a} \rightarrow \Delta_{b}$.

First we prove that it is surjective. Whenever some subset $a_{j}, a_{j+1}, \ldots, a_{j+k}$ collide, the corresponding subset $b_{j}, b_{j+1}, \ldots, b_{j+k}$ collides, because $\phi$ and $\phi^{-1}$ are normalized quasiconformal maps with maximum dilatation that depends only on $\alpha$. So the faces of $\Delta_{a}$ correspond to faces of $\Delta_{b}$ with the same names. Thus the degree of $\Phi$ must be equal to one, and the map is surjective.

In particular, this implies that for every polynomial $R$ with all roots positive and simple the full preimage under the map $W_{\alpha}$ is real, the fact which can be alternatively derived from [22, Theorem 4.3 (ii)]. It follows that the Jacobi determinant of $W_{\alpha}$ cannot be zero over a polynomial $R$ with all non-negative roots (no matter simple or not).

We have that $\Phi: \Delta_{a} \rightarrow \Delta_{b}$ has degree 1 and Jacobian in never zero. It follows that the map is a diffeomorphism. This proves the theorem.

\section{References}

[1] Q. Chen, Y. Wu and B. Xu, Developing map and character 1-form of a singular conformal metric on constant curvature one on a compact Riemann surface, arXiv:1302.6457v1.

[2] J. Dorfmeister and M. Schuster, Construction of planar CMC 4-noids of genus $\mathrm{g}=0$, JP Journal Geometry and Topology,

[3] J. Dorfmeister and J.-H. Eschenburg, Real Fuchsian equations and constant mean curvature, Matemática Contemporânea, 35 (2008), 1-25.

[4] A. Eremenko, Metrics of positive curvature with conic singularities on the sphere, Proc. Amer. Math. Soc. 132 (2004), 3349-3355 
[5] A. Eremenko and A. Gabrielov, Rational functions with real critical points and the B. and M. Shapiro conjecture in real enumerative geometry, Ann. Math., 155 (2002) 105-129.

[6] A. Eremenko and A. Gabrielov, Elementary proof of the B. and M. Shapiro conjecture for rational functions, in the book: Notions of positivity and the geometry of polynomials, trends in mathematics, Springer, Basel, 2011, p. 167-178.

[7] A. Eremenko and A. Gabrielov, Counterexamples to pole placement by static output feedback, Linear Algebra and Appl., 351-352 (2002) 211218.

[8] A. Eremenko, A. Gabrielov, M. Shapiro and A. Vainshtein, Rational functions and real Schubert calculus, Proc. AMS, 134 (2006), no. 4, 949-957.

[9] L. Ford, Automorphic functions, NY, McGraw Hill, 1929.

[10] S. Fujimori, Y. Kawakami, M. Kokubu, W. Rossman, M. Umehara and K. Yamada, CMC-1 trinoids in hyperbolic 3-space and metrics of constant curvature one with conical singularities on the 2-sphere, Proc. Japan Acad., 87 (2011), 144-149.

[11] M. Gaudin, La fonction d'onde de Bethe, Masson, Paris, 1983

[12] L. Goldberg, Catalan numbers and branched coverings by the Riemann sphere, Adv. Math. 85 (1991), no. 2, 129-144.

[13] W. Golubew, Vorlesungen über Differentialgleichungen im Komplexen, VEB Deutscher Verlag der Wissenschaften, Berlin 1958.

[14] E. Hilb, Über Kleinsche Theoreme in der Theorie der linearen Differentialgleichingen, Ann. Math., 66 (1908) 215-257.

[15] E. Hilb, Über Kleinsche Theoreme in der Theorie der linearen Differentialgleichingen, Ann. Math., 68 (1910) 24-74.

[16] C. Hodgson, I. Rivin, A characterization of compact convex polyhedra in hyperbolic 3-space, Invent. Math. 111 (1993), no. 1, 77-111. 
[17] F. Klein, Ueber die Nullstellen der hypergeometrischen Reihe, Math. Ann., 37 (1890) 573-590.

[18] F. Klein, Bemerkungen zur Theorie der linearen Differentialgleichungen zweiter Ordnung, Math. Ann., 64 (1907) 176-196.

[19] F. Luo and G. Tian, Liouville equation and spherical convex polytopes, Proc. Amer. Math. Soc. 116 (1992), no. 4, 1119-1129.

[20] R. McOwen, Point singularities and conformal metrics on Riemann surfaces, Proc. Amer. Math. Soc. 103 (1988), 222-224.

[21] E. Mukhin, V. Tarasov and A. Varchenko, The B. and M. Shapiro conjecture in real algebraic geometry and the Bethe ansatz, Ann. of Math. (2) 170 (2009), no. 2, 863-881.

[22] E. Mukhin. E. Tarasov and A. Varchenko, On reality property of Wronski maps, Confluentes Math. 1 (2009), no. 2, 225-247.

[23] E. Mukhin. E. Tarasov and A. Varchenko, Schubert calculus and representations of the general linear group, J. Amer. Math. Soc. 22 (2009) no. 4, 909-940.

[24] E. Picard, De l'equation $\Delta u=k e^{u}$ sur une surface de Riemann fermée, J. Math. Pures Appl 9 (1893) 273-292.

[25] E. Picard, De l'equation $\Delta u=e^{u}$, J. Math Pures Appl., 4 (1898) 313316.

[26] E. Picard, De l'integration de l'equation $\Delta u=e^{u}$ sur une surface de Reimann fermée, J. reine angew. Math., 130 (1905) 243-258.

[27] E. Picard, Quelques applications analytiques de la théorie des courbes et des surfaces algébriques, Gauthier-Villars, Paris, 1931.

[28] H. Poincaré, Les fonctions Fuchsiennes et l'équation $\Delta u=e^{u}$, J. Math. pures et appl., 4 (1898) 137-230. 8 (1944) 243-280.

[29] A. Ronveaux, ed., Heun's differential equations, Oxford Univ. Press, NY, 1995. 
[30] H. P. de Saint-Gervais, Uniformisation des surfaces de Riemann, ENS Éditions, 2010.

[31] I. Scherbak, Rational functions with prescribed critical points, Geom. Funct. Anal. 12 (2002), no. 6, 1365-1380.

[32] V. I. Smirnov, The problem of inversion of a linear differential equation of second order with four singularities, Petrograd, 1918 (Russian). Reproduced in V. I Smirnov, Selected works, vol. 2, Analytic theory of ordinary differential equations, St. Peterburg University, St. Peterburg, 1996.

[33] V. Smirnoff, Sur les équations différentialles linéaires du second ordre et la théorie des fonctions automorphes, Bull. Sci. Math., 45 (1921) 93120, 126-135. topological aspects of a class of mean field equations on surfaces, Discrete Contin. Dyn. Syst. 28 (2010), no. 3, 931.973.

[34] M. Troyanov, Prescribing curvature on compact surfaces with conical singularities, Trans. Amer. Math. Soc., 324 (1991) 793-821.

[35] M. Troyanov, Metrics of constant curvature on a sphere with two conical singularities, Differential geometry (Peñíscola, 1988), 296-06, Lecture Notes in Math., 1410, Springer, Berlin, 1989.

[36] M. Umehara and K. Yamada, Metrics of constant curvature 1 with three conical singularities on the 2-sphere, Illinois J. Math., 44, 1 (2000) 72 94.

[37] M. Yoshida, A naive-topological study of the contiguity relations for hypergeometric function, Banach Center Publ., PAN, Warsawa 2005, $257-268$.

A. E. and A. G.: Department of Mathematics, Purdue University, West Lafayette, IN 47907-2067 USA

V. T.: Department of Mathematics, IUPUI,

Indianapolis, IN 46202-3216 USA;

St. Petersburg branch of Steklov Mathematical Institute, 